\title{
POISSON SUPERPOSITION PROCESSES
}

\author{
HARRY CRANE, ${ }^{*}$ Rutgers University \\ PETER MCCULLAGH, ${ }^{* *}$ University of Chicago
}

\begin{abstract}
Superposition is a mapping on point configurations that sends the $n$-tuple $\left(x_{1}, \ldots, x_{n}\right) \in$ $X^{n}$ into the $n$-point configuration $\left\{x_{1}, \ldots, x_{n}\right\} \subset X$, counted with multiplicity. It is an additive set operation such that the superposition of a $k$-point configuration in $X^{n}$ is a $k n$-point configuration in $X$. A Poisson superposition process is the superposition in $X$ of a Poisson process in the space of finite-length $X$-valued sequences. From properties of Poisson processes as well as some algebraic properties of formal power series, we obtain an explicit expression for the Janossy measure of Poisson superposition processes, and we study their law under domain restriction. Examples of well-known Poisson superposition processes include compound Poisson, negative binomial, and permanental (boson) processes.
\end{abstract}

Keywords: Poisson point process; permanental process; compound Poisson process; negative binomial distribution; Poisson superposition

2010 Mathematics Subject Classification: Primary 60G55

\section{Introduction}

A Poisson process (PP) $\boldsymbol{X}$ on a space $\mathcal{X}$ is a random point configuration for which the counts of points in nonoverlapping subsets are independent Poisson random variables. The law of any Poisson process $X$ is determined by a mean measure $\zeta$ on $X$ so that $X(A):=\#(X \cap A)$, the number of points of $X$ in $A \subseteq X$, is a Poisson random variable with mean $\zeta(A)$. The class of $C o x$ processes generalizes Poisson processes by allowing the mean measure to arise from a random process. Cox processes are useful models in various applications, including neuroscience, finance, and quantum mechanics; see Cox and Isham (1980). Boson, or permanental, point processes appear primarily in quantum mechanical applications. Unlike the Poisson process, permanental processes incorporate nontrivial dependence between counts in nonoverlapping regions of $\mathcal{X}$, and so are more appropriate in settings with interaction between points; see Hough et al. (2006) or McCullagh and Møller (2006).

In this paper we study a class of point processes related to each of the above processes. A Poisson superposition process (PSP) is a random point configuration in $X$ obtained from a Poisson process $X$ in the space $\operatorname{seq}(X):=\bigcup_{n \geq 0} X^{n}$ of finite-length $X$-valued sequences by counting each component of each point in $X$ with its multiplicity. The projected configuration $Z$ can be regarded as either a random multiset of $X$ or a random integer-valued measure on $X$ for which $Z(\{x\})=0,1,2, \ldots$ For example, the superposition of $X=\{(2),(3),(1,3),(2,3,4)\}$ is the multiset $Z=\{1,2,2,3,3,3,4\}$ that counts each component with its multiplicity.

\footnotetext{
Received 11 March 2014; revision received 18 September 2014.

* Postal address: Department of Statistics, Rutgers University, 110 Frelinghuysen Road, Piscataway, NJ 08854, USA.

Email address: hcrane@stat.rutgers.edu

** Postal address: Department of Statistics, University of Chicago, Eckhart Hall, 5734 S. University Avenue, Chicago,

IL 60637, USA.
} 
Many well-known point processes correspond to Poisson superposition processes with a suitable mean measure, for example, compound Poisson, negative binomial, and permanental processes.

Our main theorems characterize certain structural properties of Poisson superpositions. In particular, when the mean measure $\zeta$ of the associated Poisson process has finite mass, we obtain an exact expression for the Janossy measure as a function of $\zeta$ (Theorem 4.1). Conversely, given the Janossy measure of the Poisson superposition process, we can recover the mean measure of the corresponding Poisson process (Theorem 4.2). If the mean measure has infinite mass, then both the Poisson process and the superposition process are almost surely infinite, in which case the superposition distribution is determined by its restrictions to subsets for which the number of points is finite with probability 1 . The restricted process is also a Poisson superposition, but the associated mean measure is not obtained by simple restriction of $\zeta$ to $\operatorname{seq}(A) \subset \operatorname{seq}(X)$ (Theorem 4.3).

We organize this paper as follows. In Section 2 we review some preliminary properties of scalar Poisson superposition and Poisson point processes. In Section 3 we discuss measures on finite-length sequences and prepare our discussion of Poisson superposition processes in Section 4. In Section 5 we conclude with some familiar special cases of Poisson superposition processes.

\section{Preliminaries: Poisson point processes}

Throughout this paper, $X$ is a Polish space with sufficient furnishings to accommodate a Poisson point process, i.e. $X$ has a Borel $\sigma$-field $B_{X}$ containing singletons on which a nonnegative Kingman-countable measure is defined; see Kingman (1993, Section 2.2). Foreshadowing our study of Poisson processes and their superpositions, we record some facts about Poisson random variables and scalar superpositions.

\subsection{Scalar Poisson superposition}

A random variable $X$ taking values on the nonnegative integers $\mathbb{Z}_{+}:=\{0,1,2, \ldots\}$ has the Poisson distribution with parameter $\lambda>0$, denoted $X \sim \operatorname{Po}(\lambda)$, if

$$
\mathbb{P}\{X=k\}=\frac{\lambda^{k} \mathrm{e}^{-\lambda}}{k !}, \quad k=0,1,2, \ldots
$$

The moment generating function of $X \sim \operatorname{Po}(\lambda)$ is $M_{\lambda}(t):=\exp \left\{\lambda\left(\mathrm{e}^{t}-1\right)\right\}$, from which infinite divisibility and the convolution property are evident, i.e. the sum $X .:=\sum_{r} X_{r}$ of independent Poisson random variables $X_{r} \sim \operatorname{Po}\left(\lambda_{r}\right)$ is distributed as $X . \sim \operatorname{Po}\left(\sum_{r} \lambda_{r}\right)$.

Definition 2.1. (Scalar Poisson superposition.) For a family $\left\{X_{r}\right\}_{r \geq 1}$ of independent Poisson random variables, with $X_{r} \sim \operatorname{Po}\left(\lambda_{r}\right)$ for each $r \geq 1$, we call

$$
Z:=\sum_{r \geq 1} r X_{r}
$$

a scalar Poisson superposition with parameter $\lambda=\left\{\lambda_{r}\right\}_{r \geq 1}$.

Note that $Z$ lives on the extended nonnegative integers $\{0,1, \ldots, \infty\}$, but, unlike the Poisson distribution, $\mathbb{E}(Z)=\infty$ does not imply $Z=\infty$ with probability 1 . For example, if $\lambda_{r}=1 / r^{2}$, then $\mathbb{E}\left(X_{r}\right)=1 / r^{2}$ and $\mathbb{E}(Z)=\sum_{r \geq 1} r \cdot 1 / r^{2}=\infty$, but $\mathbb{P}\left\{X_{r}>0\right\}=1-\mathrm{e}^{-1 / r^{2}}$ is summable. The Borel-Cantelli Lemma implies that $\mathbb{P}\left\{X_{r}>0\right.$ infinitely often $\}=0$ and $Z<\infty$ with probability 1 . 
In the next theorem, we write $\lambda(t):=\sum_{r \geq 1} t^{r} \lambda_{r}$ for the ordinary generating function of $\left\{\lambda_{r}\right\}_{r \geq 1}$.

Theorem 2.1. Scalar Poisson superpositions satisfy the following.

- Convolution. Let $Z, Z^{\prime}$ be independent scalar Poisson superpositions with parameters $\left\{\lambda_{r}\right\}$ and $\left\{\lambda_{r}^{\prime}\right\}$, respectively. Then $Z+Z^{\prime}$ is a scalar Poisson superposition with parameter $\left\{\lambda_{r}+\lambda_{r}^{\prime}\right\}$

- Infinite divisibility. For each $n \geq 1$, a scalar Poisson superposition with parameter $\left\{\lambda_{r}\right\}$ can be expressed as the sum of $n$ independent and identically distributed (i.i.d.) scalar Poisson superpositions with parameter $\left\{\lambda_{r} / n\right\}$.

- Nonnegative integer multiplication. For $m \geq 0, m Z$ has probability generating function $(P G F) \exp \left\{-\lambda(1)+\lambda\left(t^{m}\right)\right\}$.

- Superposition of scalar Poisson superpositions. If $Z_{1}, Z_{2}, \ldots$ are independent scalar Poisson superpositions, $Z_{j}$ with parameter $\left\{\lambda_{r}^{j}\right\}_{r \geq 1}$ for each $j=1,2, \ldots$, then

$$
\sum_{r \geq 1} r Z_{r}
$$

is a scalar Poisson superposition with parameter $\left\{\lambda_{r}^{*}\right\}_{r \geq 1}$, where

$$
\lambda_{r}^{*}:=\sum_{s \mid r} \lambda_{s}^{r / s},
$$

where $s \mid r$ denotes that $r=s k$ for some $k=1,2, \ldots$

- Thinning. Let $X_{1}, X_{2}, \ldots$ be independent Poisson random variables with $X_{r} \sim \operatorname{Po}\left(\lambda_{r}\right)$ and, given $\left\{X_{r}\right\}_{r \geq 1}, B_{1}, B_{2}, \ldots$ are conditionally independent binomial random variables with $B_{r} \sim \operatorname{bin}\left(X_{r}, p\right)$ for some $0<p<1$. Then the thinned superposition $Z_{p}:=\sum_{r \geq 0} r B_{r}$ is a scalar Poisson superposition with parameter $\left\{p \lambda_{r}\right\}_{r \geq 1}$.

Proof. Let $\left\{X_{r}\right\}_{r \geq 1}$ be independent Poisson random variables with $X_{r} \sim \operatorname{Po}\left(\lambda_{r}\right)$ for each $r \geq 1$. By the convolution property, the sum $X .:=\sum_{r \geq 1} X_{r}$ is a Poisson random variable with parameter $\lambda(1)=\sum_{r \geq 1} \lambda_{r}$. If $\lambda(1)=\infty$ then $X .=Z=\infty$ with probability 1 . Otherwise, if $\lambda(1)<\infty$, then the joint PGF of $(X ., Z)$ is

$$
\begin{aligned}
G(s, t) & :=\sum_{i, j \geq 0} \mathbb{P}\{X .=i, Z=j\} s^{i} t^{j} \\
& =\prod_{r \geq 1} \sum_{j \geq 0} \frac{\mathrm{e}^{-\lambda_{r}} \lambda_{r}^{j} s^{j} t^{r j}}{j !} \\
& =\prod_{r \geq 1} \mathrm{e}^{-\lambda_{r}} \mathrm{e}^{s t^{r} \lambda_{r}} \\
& =\exp \{-\lambda(1)+s \lambda(t)\} .
\end{aligned}
$$

From $G(s, t)$, we can immediately recover the marginal generating functions of $X$. and $Z$ : $G_{X,}(s)=\exp \{-\lambda(1)+s \lambda(1)\}$ and $G_{Z}(t)=\exp \{-\lambda(1)+\lambda(t)\}$.

The above properties are now immediate by standard properties of Poisson random variables. For $Z, Z^{\prime}$ independent scalar Poisson superpositions with parameters $\left\{\lambda_{r}\right\},\left\{\lambda_{r}^{\prime}\right\}$, respectively, 
we have $G_{Z+Z^{\prime}}(t)=G_{Z}(t) G_{Z^{\prime}}(t)=\exp \left\{-\lambda(1)+\lambda(t)-\lambda^{\prime}(1)+\lambda^{\prime}(t)\right\}$. To observe infinite divisibility of $Z$ with parameter $\lambda$, we simply take $Z_{1}, \ldots, Z_{n}$ i.i.d. Poisson superpositions with parameter $\left\{\lambda_{r} / n\right\}$ and appeal to the convolution property. The transformation of $G_{Z}(t)$ under nonnegative integer scaling of $Z$ follows by substituting $t^{m}$ for $t$ in $G_{Z}(t)$. Finally, let $Z_{1}, Z_{2}, \ldots$ be independent scalar Poisson superpositions, where $Z_{j}$ has parameter $\left\{\lambda_{r}^{j}\right\}_{r \geq 1}$. Then $\sum_{r \geq 1} r Z_{r}$ is also a scalar Poisson superposition with parameter $\left\{\lambda_{r}^{*}\right\}_{r \geq 1}$, where

$$
\lambda_{r}^{*}:=\sum_{s \mid r} \lambda_{s}^{r / s}
$$

The thinning property follows because the unconditional distribution of $B_{r}$ is $\operatorname{Po}\left(\lambda_{r}\right)$ for each $r=1,2, \ldots$, and $X_{1}, X_{2}, \ldots$ are independent by assumption. This completes the proof.

Example 2.1. (Compound Poisson distribution.) The compound Poisson distribution is defined as a convolution of a Poisson random number of integer-valued random variables. Let $N \sim$ $\operatorname{Po}(\mu)$ and $X_{1}, X_{2}, \ldots$ be i.i.d. according to $p_{k}:=\mathbb{P}\left\{X_{1}=k\right\}, k=0,1,2, \ldots$ The random variable $W=X_{1}+\cdots+X_{N}$ has the compound Poisson distribution with PGF

$$
G_{W}(t)=\exp \left\{\mu\left(\phi_{X}(t)-1\right)\right\}
$$

where

$$
\phi_{X}(t):=\sum_{k \geq 0} p_{k} t^{k}
$$

is the PGF of each $X_{i}, i=1,2, \ldots$ With $\lambda(t):=\mu \phi_{X}(t)$, we observe that

$$
G_{W}(t)=\exp \{-\lambda(1)+\lambda(t)\},
$$

which is also the PGF of a scalar Poisson superposition with parameter $\left\{\lambda_{r}\right\}_{r \geq 1}$, as in (2.1).

2.1.1. Relation to the negative binomial distribution. A special case of scalar Poisson superposition that occurs in applications takes $\lambda_{r}=\theta \pi^{r} / r$, with $0<\pi<1$ and $\theta>0$, for each $r \geq 1$. In this instance, we obtain $\lambda(t)=-\theta \log (1-\pi t)$ and $G_{Z}(t)=(1-\pi)^{\theta}(1-\pi t)^{-\theta}$, the generating function of the negative binomial distribution with parameter $(\theta, \pi)$. This elementary form of univariate Poisson superposition arises in diverse areas, including ecology (Fisher $e t$ al. (1943)), population genetics (Ewens (1972)), probabilistic number theory (Donnelly and Grimmett (1993)), and theory of exchangeable random partitions (Kingman (1978)).

For example, if the initial Poisson sequence is written in integer partition style, i.e. $X=$ $1^{X_{1}} 2^{X_{2}} \ldots$, where $X_{r}$ counts the number of parts of size $r$ in a partition of the random integer $Z=\sum_{r} r X_{r}$, then $X$ partitions $Z$ into a random number $X$. of parts with joint distribution in (2.1). In particular, the conditional distribution of $X$, given $Z$, is

$$
\mathbb{P}\left\{X=\left(x_{1}, x_{2}, \ldots\right) \mid Z=n\right\} \propto \prod_{1 \leq r \leq n} \frac{\lambda_{r}^{x_{r}}}{x_{r} !}, \quad \sum_{r \geq 1} r x_{r}=n .
$$

When the intensity sequence has the negative binomial pattern, $\lambda_{r}=\theta \pi^{r} / r$, then the conditional distribution is independent of $\pi$,

$$
\mathbb{P}\left\{X=\left(x_{1}, x_{2}, \ldots\right) \mid Z=n\right\}=\frac{n ! \theta^{\sum x_{r}}}{\theta^{\uparrow n} \prod_{j} j^{x_{j}} x_{j} !},
$$

where $\theta^{\uparrow n}:=\theta(\theta+1) \cdots(\theta+n-1)$ is the ascending factorial. Equation (2.2) is the Ewens sampling formula with parameter $\theta>0$ on the space of partitions of the integer $n$; see Ewens (1972) and Arratia et al. (1992). 
2.1.2. Composition of formal power series. The statistical properties in Theorem 2.1 are related to the algebraic properties of the space of formal power series generated by the monomials $t, t^{2}, t^{3}, \ldots$ Consider functions $f$ and $g$ with $f(0)=g(0)=0$ and $n$th Taylor coefficients $f_{n}$ and $g_{n}$, respectively, so that $f(t)=\sum_{n \geq 1} f_{n} t^{n} / n$ !. Then the $n$th Taylor coefficient of the composition $(f g)(t):=f(g(t))$ is

$$
(f g)_{n}=\sum_{\pi \in \mathcal{P}_{n}} f_{\# \pi} \prod_{b \in \pi} g_{\# b},
$$

where $\pi \in \mathcal{P}_{n}$ is a set partition of $[n]:=\{1, \ldots, n\}$ and \# $\pi$ equals the number of blocks of $\pi$.

The exponential equation (2.3) is related to the lifting of (2.2) from integer partitions to set partitions by sampling uniformly among set partitions whose block sizes correspond to the parts of a random integer partition drawn from (2.2). Taking $f_{n}:=\theta^{n}$ and $g_{n}:=(n-1)$ !, so that $f(t)=\mathrm{e}^{\theta t}-1$ and $g(t)=-\log (1-t)$, the coefficient in (2.3) gives the normalizing constant $\theta^{\uparrow n}$ in the Ewens distribution on set partitions,

$$
\mathbb{P}_{n}^{\theta}\{\pi\}:=\frac{\theta^{\# \pi}}{\theta^{\uparrow n}} \prod_{b \in \pi}(\# b-1) !, \quad \pi \in \mathcal{P}_{n} .
$$

More generally, the two-parameter Ewens-Pitman $(\alpha, \theta)$ distribution on $\mathcal{P}_{n}$ is defined by

$$
\mathbb{P}_{n}^{\alpha, \theta}\{\pi\}:=\frac{(\theta / \alpha)^{\uparrow \# \pi}}{\theta^{\uparrow n}} \prod_{b \in \pi}-(-\alpha)^{\uparrow \# b}, \quad \pi \in \mathcal{P}_{n},
$$

where $(\alpha, \theta)$ satisfies either

- $\alpha<0$ and $\theta=-\alpha \kappa$ for $\kappa=1,2, \ldots$ or

- $0 \leq \alpha \leq 1$ and $\theta>-\alpha$.

By putting $f_{n}:=(\theta / \alpha)^{\uparrow n}$ and $g_{n}:=-(-\alpha)^{\uparrow n}$, the composition (2.3) again gives the normalizing constant $\theta^{\uparrow n}$ in (2.5). The Ewens distribution (2.4) with parameter $\theta$ coincides with the Ewens-Pitman distribution with parameter $(0, \theta)$.

The connection between (2.3) and (2.5) is curious in a few respects. On the one hand, (2.3) is an elementary algebraic property of exponential generating functions. On the other hand, the Ewens-Pitman distribution arises in various contexts and, in fact, characterizes the class of canonical Gibbs ensembles on partitions of [ $n]$; see Pitman (2006).

2.1.3. Relation to the $\alpha$-permanent. The connection between the Ewens distribution and coefficients of formal power series is also related to the algebraic properties of the $\alpha$-permanent, which arises in theoretical computer science (Valiant $(1979,(\alpha=1))$ ), statistics (Vere-Jones (1997), (1998), Rubak et al. (2010)), and stochastic processes (Crane (2013a), Macchi (1975), and McCullagh and Møller (2006)). For $\alpha \in \mathbb{R}$ and an $\mathbb{R}$-valued matrix $M:=\left(M_{i j}\right)_{1 \leq i, j \leq n}$, the $\alpha$-permanent of $M$ is defined by

$$
\operatorname{per}_{\alpha}(M):=\sum_{\sigma \in \delta_{n}} \alpha^{\# \sigma} \prod_{j=1}^{n} M_{j, \sigma(j)},
$$

where the sum is over the symmetric group $\delta_{n}$ of permutations of $[n]$ and $\# \sigma$ denotes the number of cycles of $\sigma \in \S_{n}$. To show the connection to (2.3), we appeal to the identity

$$
\operatorname{per}_{\alpha \beta}(M)=\sum_{\pi \in \mathcal{P}_{n}} \beta^{\downarrow \# \pi} \prod_{b \in \pi} \operatorname{per}_{\alpha}(M[b])
$$


where $\beta^{\downarrow j}:=\beta(\beta-1) \cdots(\beta-j+1)$ and $M[b]:=\left(M_{i j}\right)_{i, j \in b}$ is the submatrix of $M$ indexed by $b \subseteq[n]$; see Crane (2013b). When $M:=J_{n}$ is the $n \times n$ matrix of all 1 s, this identity simplifies to

$$
(\alpha \beta)^{\uparrow n}=\sum_{\pi \in \mathcal{P}_{n}} \beta^{\downarrow \# \pi} \prod_{b \in \pi} \alpha^{\uparrow \# b},
$$

from which the two-parameter distribution (2.5) is apparent by the transformation $\beta \mapsto \theta / \alpha$.

\subsection{Janossy measures}

The law of any finite random point configuration $X \subseteq X$ is determined by the Janossy measure $J=\sum_{n \geq 1} J^{(n)}$, where each $J^{(n)}$ is a nonnegative symmetric measure on $X^{n}$ such that $\mathbb{P}\{\# \boldsymbol{X}=n\} \stackrel{=}{=} J^{(n)}\left(X^{n}\right)$ and $\sum_{n \geq 1} J^{(n)}\left(X^{n}\right)=1$. Each orbit of the symmetric group acting on $X^{n}$ corresponds to an $n$-point configuration in $X$, so we can identify $J$ with a probability measure on the space of finite point configurations. Equivalently, each symmetric subset $A \subseteq X^{n}$ determines a set of $n$-point configurations and, in some cases, it is convenient to define the configuration measures $\left\{J_{n}\right\}_{n \geq 1}$ by

$$
J_{n}(A)=\sum_{\sigma \in \delta_{n}} J^{(n)}(\sigma A)=n ! J^{(n)}(A)
$$

where $\sigma A=\left\{\left(x_{\sigma(1)}, \ldots, x_{\sigma(n)}\right): x \in A\right\}$ denotes the image of $A$ by relabeling coordinates according to $\sigma$.

The configuration measures of a Poisson process with finite mean measure $\zeta$ are

$$
J_{n}\left(\mathrm{~d} x_{1} \cdots \mathrm{d} x_{n}\right)=\mathrm{e}^{-\zeta} \cdot \prod_{j=1}^{n} \zeta\left(\mathrm{d} x_{j}\right), \quad n=0,1, \ldots
$$

where $\zeta_{\bullet}=\zeta(X)$. Thus, $J^{(n)}\left(X^{n}\right)=\mathrm{e}^{-\zeta_{\bullet}} \zeta_{\bullet}^{n} / n$ ! is the probability that $\# \boldsymbol{X}=n$.

Remark 2.1. (Notation.) Consistent with our notation for Janossy measures, we adopt the following convention for measures on $\operatorname{seq}(\mathcal{X})$. Throughout the paper, $\zeta$ denotes the mean measure for a Poisson point process on $\operatorname{seq}(X)$, whose restriction $\zeta^{(n)}$ to $X^{n}$ need not be symmetric. The symmetrized version is

$$
\zeta_{n}(A):=\sum_{\sigma \in \delta_{n}} \zeta^{(n)}(\sigma A), \quad A \subseteq X^{n}
$$

Each Janossy component measure $J^{(n)}$ is automatically symmetric on $X^{n}$, but the configuration measures $\left\{J_{n}\right\}_{n \geq 1}$ in (2.6) are more natural for Poisson superposition processes. If $A \subseteq X^{n}$ is symmetric, i.e. $A=\sigma A$ for all $\sigma \in \S_{n}$, then $J^{(n)}(A)=J_{n}(A) / n$ !.

\section{Finite sequences}

Superposition sends each point $\boldsymbol{x}=\left(x_{1}, \ldots, x_{n}\right) \in X^{n}$ to the $n$-point configuration $\operatorname{Spp}(\boldsymbol{x})=\left\{x_{1}, \ldots, x_{n}\right\} \subset \mathcal{X}$, that is, the multiset whose elements are the components of $\boldsymbol{x}$ counted with multiplicity. More generally, the superposition of the $k$-point configuration $\left\{x_{1}, \ldots, x_{k}\right\} \subset \operatorname{seq}(X)$ is the additive multiset operation defined as the union of the superpositions, counted with multiplicity. 
Definition 3.1. (Poisson superposition process, version 1.) If $X \sim \operatorname{PP}(\zeta)$ is a Poisson point process in $\operatorname{seq}(X)$, then $Z=\operatorname{Spp}(X)$ is a Poisson superposition process in $X$. In words, $\boldsymbol{X}=\left(\boldsymbol{X}_{0}, \boldsymbol{X}_{1}, \ldots\right)$ is a sequence of independent Poisson processes $\boldsymbol{X}_{r} \sim \operatorname{PP}\left(\zeta^{(r)}\right)$ on $X^{r}$ and $Z$ records the components of every point in $X$ with multiplicity.

For the remainder of the paper, we equip seq $(X)$ with the product $\sigma$-field $\bigotimes_{n \geq 0} \mathscr{B}_{X}^{n}$, where $B_{X}^{n}:=B_{X} \otimes \cdots \otimes B_{X}$ is the $n$-fold product of Borel $\sigma$-fields on $X$.

We formalize Definition 3.1 through the following discussion of embedded subsequences.

\subsection{Embedded subsequences}

For $n \geq 1$, let $\boldsymbol{x}:=\left(x_{1}, \ldots, x_{n}\right) \in \mathcal{X}^{n}$ be a length- $n$ sequence with components in $\mathcal{X}$. Each ordered subset of integers $i:=\left(i_{1}, \ldots, i_{m}\right)$ for which $1 \leq i_{1}<\cdots<i_{m} \leq n$ determines an embedded length- $m$ subsequence $x[i]:=\left(x_{i_{1}}, \ldots, x_{i_{m}}\right)$. There may be multiple ways to embed a length- $m$ sequence into a length- $n$ sequence, $m \leq n$, and so we define the embedding number

$$
L\left(\boldsymbol{x}^{\prime}, \boldsymbol{x}\right):=\#\left\{i=\left(i_{1}, \ldots, i_{m}\right): 1 \leq i_{1}<\cdots<i_{m} \leq n, \boldsymbol{x}[i]=\boldsymbol{x}^{\prime}\right\}
$$

as the number of ways to embed $\boldsymbol{x}^{\prime}$ in $\boldsymbol{x}$. From (3.1), we define the embedding measure

$$
L(A, \boldsymbol{x}):=\sum_{\boldsymbol{x}^{\prime} \in A} L\left(\boldsymbol{x}^{\prime}, \boldsymbol{x}\right), \quad A \subseteq \operatorname{seq}(\mathcal{X}),
$$

which counts the number of embedded subsequences of $x$ contained in $A$, with the convention $L\left(X^{0}, x\right)=1$ for all $x \in \operatorname{seq}(X)$. For fixed $x \in \operatorname{seq}(X), L(\cdot, x)$ is a finite measure on $\operatorname{seq}(X)$ with total mass

$$
L(\operatorname{seq}(X), x):=\sum_{m \geq 0}\left(\begin{array}{c}
\# x \\
m
\end{array}\right)=2^{\# x},
$$

where $\# \boldsymbol{x}$ denotes the length of $\boldsymbol{x}$.

For $\boldsymbol{x} \in \operatorname{seq}(\mathcal{X})$, we define the first-order superposition $\operatorname{Spp}(\boldsymbol{x})$ of $\boldsymbol{x}$ to be the multiset whose elements are all length- 1 subsequences (with multiplicity) embedded in $\boldsymbol{x}$. Formally, $\operatorname{Spp}(\boldsymbol{x})$ is determined by the restriction of $L(\cdot, x)$ to Borel subsets of $\mathcal{X}$, where $L(y, x)$ counts the number of occurrences of $y \in X$ in $\boldsymbol{x}$. For $m \geq 1$, we write $L^{(m)}(\cdot, \boldsymbol{x})$ to denote the restriction of the embedding measure $L(\cdot, \boldsymbol{x})$ to Borel subsets of $X^{m}$.

Definition 3.2. (Poisson superposition process, version 2.) Let $\boldsymbol{X}$ be a Poisson process with mean measure $\zeta$ on $\operatorname{seq}(X)$. The Poisson superposition process $Z \sim \operatorname{PSP}(\zeta)$ is the random measure obtained by first-order superposition of $\boldsymbol{X}$,

$$
Z(A):=L^{(1)}(A, X) \text { for } A \subset X .
$$

The mean measure or first-order intensity is $\kappa(A)=\mathbb{E}(Z(A))$.

In the following example we show that the preceding definition by embedding coincides with the description from the beginning of Section 3.

Example 3.1. Let $X=\mathbb{Z}$ and suppose that

$$
\boldsymbol{X}_{1}=\{(1),(3)\}, \quad \boldsymbol{X}_{2}=\{(2,0),(1,2),(3,4)\}, \quad \boldsymbol{X}_{3}=\{(1,2,1)\},
$$

and $\boldsymbol{X}_{n}=\varnothing, n \geq 4$, are realizations of independent Poisson processes on $\chi^{1}, X^{2}, \ldots$, respectively. Then the superposition on $X$ is the multiset $Z=\left\{0^{1}, 1^{4}, 2^{3}, 3^{2}, 4^{1}\right\}$, i.e. $Z(0)=1$, $Z(1)=4, Z(2)=3, Z(3)=2, Z(4)=1$, and $Z(x)=0$ otherwise. 
By our phrasing in terms of the embedding measure, we can exploit properties of measures on finite sequences to study Poisson superposition processes. The following sections, though technical, are necessary to prove our main theorems.

\subsection{Measures on finite sequences}

For any measure $\zeta$ on seq $(X)$, we define the measure $L \zeta$ on seq $(X)$ as the mixture of $L(A, \cdot)$ with respect to $\zeta$,

$$
(L \zeta)(A):=\int_{\operatorname{seq}(x)} L(A, x) \mathrm{d} \zeta(x), \quad A \subseteq \operatorname{seq}(X)
$$

As $\operatorname{seq}(X)$ is a disjoint union of $X^{0}, X^{1}, X^{2}, \ldots$, each measure $\zeta$ on seq $(X)$ decomposes as the sum of its disjoint component measures $\zeta=\sum_{r \geq 0} \zeta^{(r)}$. Moreover, when restricted to the subset of length- $m$ sequences, we define

$$
L \zeta^{(m)}(A):=\int_{\operatorname{seq}(X)} L(A, x) \mathrm{d} \zeta^{(m)}(x)=\int_{X^{m}} L(A, x) \mathrm{d} \zeta(x) .
$$

Therefore, we can also write $L \zeta=\sum_{r \geq 0} L \zeta^{(r)}$, but the components $\left\{L \zeta^{(r)}\right\}_{r \geq 1}$ need not be mutually singular.

3.2.1. Superposition and disaggregation measures. A measurable subset $A \subseteq X$ acts linearly on $\zeta=\sum_{r \geq 0} \zeta^{(r)}$ by transforming each component measure $\zeta^{(r)} \mapsto A \zeta^{(r)}$ in the obvious way: for each $n=1,2, \ldots$,

$$
\left(A \zeta^{(n)}\right)\left(B_{1} \cdots B_{n}\right)=\sum_{r=0}^{n} \zeta^{(n+1)}\left(B_{1} \cdots B_{r} \cdot A \cdot B_{r+1} \cdots B_{n}\right)
$$

where $B_{1}, \ldots, B_{n}$ are measurable subsets of $\mathcal{X}$, and $B_{1} \cdots B_{n}$ denotes the Cartesian product. For example, $n=0,1$ correspond to $\left(A \zeta^{(0)}\right)\left(X^{0}\right)=\zeta^{(1)}(A)$ and $\left(A \zeta^{(1)}\right)(B)=\zeta^{(2)}(A \times B)+$ $\zeta^{(2)}(B \times A)$, respectively. Iterating the above action for $A_{1}, \ldots, A_{k} \subseteq X$ gives the $k$-fold action

$$
\left(A_{1} \cdots A_{k} \zeta^{(n)}\right)\left(B_{1} \cdots B_{n}\right)=\sum_{\sigma \in S_{k}} \sum_{1 \leq i_{1}<\cdots<i_{k} \leq n+k} \zeta^{(n+k)}\left(C_{1} \cdots C_{n+k}\right),
$$

where $C_{1}, \ldots, C_{n+k}$ are subsets of $\mathcal{X}$ such that $C_{i_{r}}=A_{\sigma(r)}$ and $C_{\bar{i}_{r}}=B_{r}$, where $1 \leq \bar{i}_{1}<$ $\cdots<\bar{i}_{n} \leq n+k$ is the complement of $1 \leq i_{1}<\cdots<i_{k} \leq n+k$. It follows that the action (3.3) on $\zeta$ is commutative, $A_{\sigma(1)} \cdots A_{\sigma(k)} \zeta^{(n)}=A_{1} \cdots A_{k} \zeta^{(n)}$ for all permutations $\sigma:[k] \rightarrow[k]$, but the resulting measure on $X^{n+k}$ need not be symmetric.

Choosing $A_{1}=\cdots=A_{k}=\chi$ in (3.3), yields

$$
\left(X^{k} \zeta^{(n)}\right)\left(B^{[1: n]}\right)=k ! \sum_{1 \leq i_{1}<\cdots<i_{n} \leq n+k} \zeta^{(n+k)}\left(C^{[1: n+k]}\right)
$$

where $B^{[1: n]}:=B_{1} \times \cdots \times B_{n}$ is the Cartesian product, $C_{i_{j}}=B_{j}$, and $C_{\bar{i}_{j}}=X$. For a pair of measurable sets $A_{1}, A_{2} \subset \mathcal{X}$, we extend the action in (3.2) to formal linear combinations

$$
\left(A_{1}+A_{2}\right) \zeta:=A_{1} \zeta+A_{2} \zeta
$$


from which we define the superposition measure

$$
\exp (x) \zeta:=\sum_{k \geq 0} \frac{x^{k} \zeta}{k !}=L \zeta
$$

In the reverse direction, we define the disaggregation measure of $\kappa:=L \zeta$ on $\operatorname{seq}(\mathcal{X})$ by

$$
\exp (-X) \kappa:=\sum_{k \geq 0} \frac{(-1)^{k}\left(X^{k} \kappa\right)}{k !}=\zeta
$$

The transformation $\zeta \mapsto \kappa$ is invertible. But, unlike the relation between moments and cumulants, the first $n$ components of $\zeta$ do not determine the first $n$ components of $\kappa$ and vice versa. In particular, the first-order superposition measure $\kappa^{(1)}=L \zeta^{(1)}$ does not determine $\zeta^{(1)}$.

\subsection{Subset restriction}

Janossy measures only determine the law of point processes that are almost surely finite. For infinite, but locally finite, point processes, the law is determined by restriction to certain test sets $\mathcal{V} \subset \mathcal{X}$.

On the one hand, $\mathcal{V}^{n} \subset \mathcal{X}^{n}$ implies that each $\mathcal{V}$-sequence is also an $\mathcal{X}$-sequence, so the natural embedding $\operatorname{seq}(\mathcal{V}) \hookrightarrow \operatorname{seq}(X)$ preserves sequence length and component order. On the other hand, subset restriction also determines a natural deletion map $T: \operatorname{seq}(\mathcal{X}) \rightarrow \operatorname{seq}(\mathcal{V})$ in the reverse direction, by which each component of the $X$-sequence $x=\left(x_{1}, \ldots, x_{n}\right)$ that does not belong to $\mathcal{V}$ is deleted, leaving the remaining components $T \boldsymbol{x}=\left(x_{i_{1}}, \ldots, x_{i_{k}}\right)$ in their original relative order $i_{1}<\cdots<i_{k}$. In other words, $T \boldsymbol{x}$ is the maximal embedded subsequence belonging to $\operatorname{seq}(\mathcal{V})$.

If $\zeta$ is a measure on $\operatorname{seq}(X)$, then the induced measure $T \zeta$ on seq $(\mathcal{V})$ is defined as the usual image measure

$$
(T \zeta)(A)=\zeta\left(T^{-1} A\right), \quad A \subseteq \operatorname{seq}(\mathcal{V}),
$$

where $T^{-1} A$ is the inverse image of $A$ in $\operatorname{seq}(X)$. The inverse image $T^{-1}(\{v\})$ of $v \in \mathcal{V}^{n}$ is the set of $X$-sequences that include $v$ as an embedded subsequence and have no other components belonging to $\mathcal{V}$, i.e. sets of the form $\{\boldsymbol{v}\} \times(X \backslash \mathcal{V})^{k}, k \geq 0$, with any of $\left(\begin{array}{c}n+k \\ k\end{array}\right)$ embeddings. Evidently, $T \zeta=\exp (X \backslash \mathcal{V}) \zeta=\exp (-\mathcal{V}) \exp (\mathcal{X}) \zeta$ and $\exp (\mathcal{V})(T \zeta)=\kappa$.

In words, if $\zeta$ is a measure on $\operatorname{seq}(\mathcal{X})$ with superposition $\kappa=\exp (\mathcal{X}) \zeta$, then the induced measure on $\mathcal{V}$-restricted sequences is obtained by subset restriction of $\kappa$ to $\operatorname{seq}(\mathcal{V}) \subset \operatorname{seq}(X)$, followed by disaggregation $(T \zeta)(A)=\exp (-\mathcal{V}) \kappa(A)$. The state of affairs is best described by a commutative diagram in which the projection $T$ and the pointwise restriction $R$ are maps on measures associated with the subset $\mathcal{V} \subset \mathcal{X}$; see Figure 1.

Commutativity asserts that, for each measure $\zeta$ on $\operatorname{seq}(X)$ and $\mathcal{V} \subset X$, the superposition of the projection $\mathrm{e}^{\mathcal{V}} T \zeta$ is a measure on seq( $\left.\mathcal{V}\right)$ equal to the restriction of the superposition $R \mathrm{e}^{X} \zeta$.

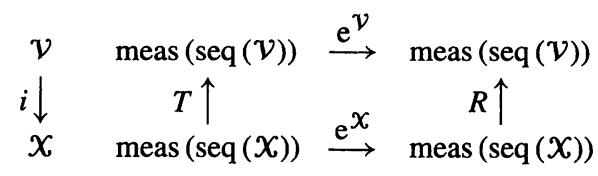

FIGURE 1: A commutative diagram. The projection $T$ and the pointwise restriction $R$ are maps on measures associated with the subset $\mathcal{V} \subset \mathcal{X}$. 


\section{Poisson superposition processes}

Let $\boldsymbol{X}_{1}, \boldsymbol{X}_{2}, \ldots$ be independent Poisson processes for which $\boldsymbol{X}_{r} \subseteq \boldsymbol{X}^{r}$ has finite mean measure $\zeta^{(r)}$ for each $r=1,2, \ldots$ We denote the union of $\boldsymbol{X}_{1}, \boldsymbol{X}_{2}, \ldots$ by $\boldsymbol{X}:=\bigcup_{r \geq 1} \boldsymbol{X}_{r} \subseteq$ $\operatorname{seq}(X)$ and their superposition in $X$ by $Z(\cdot):=L^{(1)}(\cdot, X)$, as in Definition 3.2. We further assume that $\zeta_{.}:=\sum_{r} \zeta^{(r)}\left(\mathcal{X}^{r}\right)<\infty$; whence, $n_{r}:=\# \boldsymbol{X}_{r}<\infty$ for each $r=1,2, \ldots$, and $n_{.}:=\sum_{r} n_{r}<\infty$ with probability 1 . In this case, we let $\left\{J_{r}^{(n)}\right\}_{n=1,2, \ldots}$ denote the component Janossy measures of $\left\{\boldsymbol{X}_{r}\right\}_{r \geq 1}$ and $\left\{J_{r, n}\right\}_{n=1,2, \ldots}$ be the associated configuration measures, as in (2.7). It follows that

$$
J_{r, n}\left(\mathrm{~d} x_{1}, \ldots, \mathrm{d} x_{n}\right)=\mathrm{e}^{-\zeta_{\bullet}^{(r)}} \prod_{j=1}^{n} \zeta^{(r)}\left(\mathrm{d} x_{j}\right), \quad x_{1}, \ldots, x_{n} \in X^{r},
$$

from which the component Janossy measures are given by

$$
J_{r}^{(n)}\left(\mathrm{d} x_{1}, \ldots, \mathrm{d} x_{n}\right)=(n !)^{-1} \mathrm{e}^{-\zeta_{\bullet}^{(r)}} \prod_{j=1}^{n} \zeta^{(r)}\left(\mathrm{d} x_{j}\right), \quad r \geq 1 .
$$

Theorem 4.1. Let $\boldsymbol{Z}$ be the superposition of independent Poisson processes $\boldsymbol{X}_{1}, \boldsymbol{X}_{2}, \ldots$, where each $\boldsymbol{X}_{r}$ has mean measure $\zeta^{(r)}<\infty$ on $X^{r}$ such that $\zeta_{0}:=\sum_{r \geq 1} \zeta^{(r)}\left(\mathcal{X}^{r}\right)<\infty$. Then the Janossy measures $\left\{J^{(n)}\right\}_{n \geq 0}$ of $Z$ are given by $J^{(n)}=J_{n} / n$ !, where

$$
J_{n}(\mathrm{~d} \boldsymbol{x})=\mathrm{e}^{-\zeta .} \sum_{\pi \in \mathcal{P}_{n}} \prod_{b \in \pi} \zeta_{\# b}(\mathrm{~d} \boldsymbol{x}[b]), \quad x=\left(x_{1}, \ldots, x_{n}\right) \in X^{n}, \quad n \geq 0 .
$$

Proof. Similar to (2.1), we obtain the joint probability generating functional for the Janossy measures of $\boldsymbol{X} .=\bigcup_{r \geq 1} \boldsymbol{X}_{r}$ and $\boldsymbol{Z}$ by

$$
\begin{aligned}
J(s, t) & =\prod_{r \geq 1} \sum_{j \geq 0} J_{r}^{(j)} s^{j} t^{r j} \\
& =\prod_{r \geq 1} \sum_{j \geq 0} \frac{s^{j} t^{r j}}{j !} \mathrm{e}^{-\zeta_{\bullet}^{(r)}} \prod_{i=1}^{j} \zeta^{(r)} \\
& =\mathrm{e}^{-\zeta \cdot} \prod_{r \geq 1} \sum_{j \geq 0} \frac{\left(s t^{r} \zeta^{(r)}\right)^{j}}{j !} \\
& =\exp \{-\zeta .+s \zeta(t)\}
\end{aligned}
$$

where $\zeta(t)=\sum_{r \geq 1} t^{r} \zeta^{(r)}$ is the generating functional for the mean measures $\left\{\zeta^{(r)}\right\}_{r \geq 1}$ and $\zeta^{(r)}=\zeta^{(r)}\left(X^{r}\right)$ for each $r=1,2, \ldots$ The generating functional $J(t)$ of the Janossy measures for $Z$ is, thus,

$$
J(t)=\exp \left(-\zeta_{\bullet}+\sum_{n \geq 1} t^{n} \zeta^{(n)}\right)
$$

from which (4.1) is apparent by differentiation.

Theorem 4.2. Let $Z$ be a finite point process in $\mathcal{X}$ with Janossy measure $\left\{J_{n} / n !\right\}_{n \geq 0}$ and $J_{0}>0$, and let

$$
\zeta_{n}(\mathrm{~d} \boldsymbol{x})=\sum_{\pi \in \mathcal{P}_{n}}(-1)^{\# \pi-1}(\# \pi-1) ! J_{0}^{-\# \pi} \prod_{b \in \pi} J_{\# b}(\mathrm{~d} \boldsymbol{x}[b]) \text { for } n \geq 1, \boldsymbol{x} \in X^{n} .
$$

Then $\boldsymbol{Z}$ is a Poisson superposition process if and only if $\zeta$ is nonnegative. 
Proof. The relationship (4.2) is apparent from the relationship between moments and cumulants, see McCullagh (1987, Section 2.3) or Streitberg (1990). Therefore, given a finite total measure $\zeta_{.}<\infty$ and a family of Janossy measures for a Poisson superposition process, the mean measure of the Poisson process on $\operatorname{seq}(X)$ can be recovered uniquely up to permutation of component measures.

Corollary 4.1. Let $\left\{Z_{r}\right\}_{r \geq 1}$ be independent Poisson superposition processes, where $Z_{r} \sim$ $\operatorname{PSP}\left(\zeta^{r}\right)$ for each $r=1,2, \ldots$ The convolution of $\left\{Z_{r}\right\}_{r \geq 1}$, defined by

$$
Z_{.}(\cdot):=\sum_{r \geq 1} Z_{r}(\cdot)
$$

is a Poisson superposition process with generating measure $\zeta^{\bullet}=\sum_{r} \zeta^{r}$. Moreover, every Poisson superposition process is infinitely divisible.

Proof. The proof follows from the convolution property of Poisson processes and is analogous to the proof of the corresponding property for scalar Poisson superpositions. Infinite divisibility follows immediately from the convolution property.

\subsection{Subset restriction}

Theorem 4.3. Let $Z \sim \operatorname{PSP}(\zeta)$ be a Poisson superposition process with parameter $\zeta$ on $\operatorname{seq}(X)$ and denote by $Z[\mathcal{V}]$ its restriction to $\mathcal{V} \subseteq X$. Then $Z[\mathcal{V}] \sim \operatorname{PSP}(T \zeta)$ is a Poisson superposition process on $\mathcal{V}$ with parameter $\zeta^{\mathcal{V}}:=T \zeta$ and configuration measures

$$
J_{n}^{\nu}(\mathrm{d} x)=\mathrm{e}^{-\zeta_{0}^{\nu}} \sum_{\pi \in \mathcal{P}_{n}} \prod_{b \in \pi} \zeta_{\# b}^{\nu}(\mathrm{d} x[b]), \quad x \in X^{n},
$$

where $T: \operatorname{seq}(\mathcal{X}) \rightarrow \operatorname{seq}(\mathcal{V})$ is the projection defined in Section 3.3.

Proof. To observe (4.3), we first project $\boldsymbol{X}$ to seq( $\mathcal{V})$ and then apply superposition. The projection into $\operatorname{seq}(\mathcal{V})$ remains a Poisson process since nonoverlapping subsets of $\mathcal{V}$ correspond to nonoverlapping Borel sets of $\operatorname{seq}(\mathcal{X})$ under projection; hence, counts in nonoverlapping subsets of $\operatorname{seq}(\mathcal{V})$ are independent Poisson random variables with updated intensity $\zeta^{\mathcal{V}}:=T \zeta$. This completes the proof.

\section{Special cases}

To conclude, we highlight some well-known point processes that are also Poisson superposition processes.

\subsection{Self-similar measures}

Let seq ${ }^{+}(X)$ be the set of nonnull finite sequences, $\zeta:=\sum_{n>0} \zeta^{(n)}$ be a measure on seq $(X)$, and $\mathcal{V}^{\prime} \subset \mathcal{X}$ be any subset such that

$$
\zeta\left(\operatorname{seq}^{+}\left(\mathcal{V}^{\prime}\right)\right)=\sum_{k=1}^{\infty} \zeta^{(k)}\left(\mathcal{V}^{\prime k}\right)<\infty .
$$

In other words, the restriction $\zeta\left[\mathcal{V}^{\prime}\right]$ of $\zeta$ to $\operatorname{seq}\left(\mathcal{V}^{\prime}\right) \subset \operatorname{seq}(X)$ is finite on nonnull sequences. We call $\zeta$ self-similar if, for each $\mathcal{V} \subset \mathcal{V}^{\prime} \subset \mathcal{X}$, the projection $T\left(\zeta\left[\mathcal{V}^{\prime}\right]\right)$ of the $\mathcal{V}^{\prime}$-restricted measure is proportional to the $\mathcal{V}$-restriction $\zeta[\mathcal{V}]$. Here, $\zeta[\mathcal{V}]$ is the restriction of $\zeta$ to $\operatorname{seq}(\mathcal{V}) \subset$ $\operatorname{seq}(\mathcal{X})$, while $T: \operatorname{seq}(\mathcal{X}) \rightarrow \operatorname{seq}(\mathcal{V})$ is the projection described previously. 
For a simple example, consider the weighted product measures $\zeta^{(k)}=\zeta_{1}^{\otimes k} / k$ ! on $X^{k}$ so that $\zeta\left(\operatorname{seq}\left(\mathcal{V}^{\prime}\right)\right)=\exp \left(\zeta_{1}\left(\mathcal{V}^{\prime}\right)\right)<\infty$ holds whenever $\zeta_{1}\left(\mathcal{V}^{\prime}\right)<\infty$. For $\mathcal{V} \subset \mathcal{V}^{\prime} \subset X$ such that $\zeta_{1}\left(\mathcal{V}^{\prime}\right)<\infty$, restriction of $\zeta$ to $\operatorname{seq}\left(\mathcal{V}^{\prime}\right)$ followed by projection onto $\operatorname{seq}(\mathcal{V})$ gives

$$
\left(T \zeta\left[\mathcal{V}^{\prime}\right]\right)(A)=\zeta(A) \exp \left(\zeta_{1}\left(\mathcal{V}^{\prime} \backslash \mathcal{V}\right)\right) \text { for } A \subset \operatorname{seq}(\mathcal{V})
$$

Thus, the projected measure exceeds the restricted measure by the constant factor

$$
\exp \left(\zeta_{1}\left(\mathcal{V}^{\prime} \backslash \mathcal{V}\right)\right)<\infty
$$

For a general self-similar measure $\zeta$ on $\operatorname{seq}(X)$, we denote the projection seq $\left(\mathcal{V}^{\prime}\right) \rightarrow \operatorname{seq}(\mathcal{V})$, $\mathcal{V} \subseteq \mathcal{V}^{\prime} \subseteq X$, by $T_{\mathcal{V}, \mathcal{V}^{\prime}}$. Then $T_{\mathcal{V}, \mathcal{V}^{\prime}} \zeta\left[\mathcal{V}^{\prime}\right] \propto \zeta[\mathcal{V}]$ and, in particular, $T_{\mathcal{V}} \zeta \propto \zeta[\mathcal{V}]$, where $T_{\mathcal{V}}:=T_{\mathcal{V}, x}$. The Janossy measures for the Poisson superposition process restricted to $\mathcal{V}$ are determined by (4.3), where $\zeta^{\mathcal{V}}:=T_{\mathcal{V}} \zeta$. In the self-similar case, these Janossy measures satisfy

$$
J_{n}^{\mathcal{V}}(\mathrm{d} x)=\mathrm{e}^{-\zeta .} \sum_{\pi \in \mathcal{P}_{n}} \alpha_{\mathcal{V}}^{\# \pi} \prod_{b \in \pi} \zeta_{\# b}(\mathrm{~d} x[b]), \quad x \in X^{n},
$$

where $\alpha_{\mathcal{V}}=T_{\nu} \zeta(A) / \zeta(A) \geq 1$ is the proportionality constant. Some special cases arise in this setting.

5.1.1. Product measures. As in the above example, suppose that $\zeta^{(k)}:=\zeta_{1}^{\otimes k} / k$ ! is a weighted product measure for each $k$. In this case, the symmetrized measures $\zeta_{k}=k ! \zeta^{(k)}=\zeta_{1}^{\otimes k}$ are product measures on symmetric subsets $A \subseteq X^{k}$ and (5.1) can be written as

$$
J_{n}^{\mathcal{V}}(\mathrm{d} \boldsymbol{x})=\mathrm{e}^{-\zeta \cdot} \prod_{j=1}^{n} \zeta_{1}\left(\mathrm{~d} x_{j}\right) \sum_{\pi \in \mathcal{P}_{n}} \alpha_{\mathcal{V}}^{\# \pi}=\mathrm{e}^{-\zeta} \cdot B_{n}\left(\alpha_{\mathcal{V}}\right) \prod_{j=1}^{n} \zeta_{1}\left(\mathrm{~d} x_{j}\right),
$$

where $B_{n}(\alpha):=\sum_{k} \mathcal{P}(n, k) \alpha^{k}$ is the generating function for the number of partitions of [ $n$ ] with $k$ blocks; see Wilf (2006, p. 18). Taking $\mathcal{V}=X$, we obtain

$$
J_{n}(\mathrm{~d} \boldsymbol{x})=\mathrm{e}^{-\zeta} \cdot \mathscr{B}_{n} \prod_{j=1}^{n} \zeta_{1}\left(\mathrm{~d} x_{j}\right)
$$

where $\mathcal{B}_{n}:=\# \mathcal{P}_{n}$ is the $n$th Bell number.

\subsection{Completely random superpositions}

For each $n \geq 1$, let $\zeta^{(n)}$ be diagonal, i.e. supported on the diagonal of $X^{n}$,

$$
\operatorname{diag}\left(X^{n}\right):=\left\{(x, \ldots, x) \in X^{n}: x \in X\right\} .
$$

Since $\zeta^{(n)}$ vanishes off the diagonal, it is determined by a measure $\xi^{(n)}$ on $X$ in the obvious way:

$$
\zeta^{(n)}\left(A_{1} \times \cdots \times A_{n}\right)=\xi^{(n)}\left(A_{1} \cap \cdots \cap A_{n}\right), \quad A_{i} \subset X .
$$

The first-order superposition $\boldsymbol{Z}$ is a scalar Poisson superposition in the sense that $\boldsymbol{Z}(A)=$ $\sum_{r \geq 1} r \boldsymbol{X}_{r}(A)$ is a linear combination of independent Poisson random variables with integer coefficients, as in Section 2.1. Moreover, the counts $Z(A)$ and $Z\left(A^{\prime}\right)$ are independent whenever $A$ and $A^{\prime}$ are disjoint, so each Poisson superposition $Z \sim \operatorname{PSP}(\zeta)$ is a completely random measure; see Kingman (1993, Chapter 8). 
From (5.2), the measures $\xi^{(n)}$ on $\mathcal{X}$ determine a measure $\Lambda$ on the product space $\mathbb{N} \times X$ by

$$
\Lambda(\{n\} \times A)=\xi^{(n)}(A) .
$$

If $\boldsymbol{X}$ is a Poisson process in the product space with mean measure $\Lambda$, projection into $X$ by summation of marks gives $Z(A)=\sum_{n \geq 1} n X(\{n\} \times A)$, which coincides with the diagonal Poisson superposition $\boldsymbol{Z} \sim \operatorname{PSP}(\zeta)$. Since every measure in the product space $\mathbb{N} \times X$ also determines a diagonal measure on $\operatorname{seq}(\mathcal{X})$, the argument in Kingman (1993, Section 8.2) immediately leads to the following theorem.

Theorem 5.1. A completely random integer-valued process $\mathbf{Z}(\cdot)$ on $\mathcal{X}$ with no fixed atoms is necessarily a Poisson superposition with diagonal measures.

5.2.1. Compound Poisson process. Let $N^{(1)}, N^{(2)}, \ldots$ be independent Poisson processes on $\mathbb{R}$, where $N^{(n)}$ has intensity $\lambda p_{n}$ and $\sum_{n=1}^{\infty} p_{n}=1$. Given $N^{(n)}$, we define the increment of $N^{(n)}$ by $I^{(n)}(t):=N^{(n)}(t)-N^{(n)}(t-)$ and $\boldsymbol{X}_{n}$ on $\operatorname{diag}\left(\mathbb{R}^{n}\right)$ by putting $(x, \ldots, x) \in \boldsymbol{X}_{n}$ if and only if $I^{(n)}(x)=1$. Thus, $X_{n}$ is obtained by embedding a Poisson process in $\mathbb{R}$ with intensity $\zeta^{(n)}(\mathrm{d} x)=\lambda p_{n} \mathrm{~d} x$ into $\operatorname{diag}\left(\mathbb{R}^{n}\right)$. Defining $\boldsymbol{Z}:=\operatorname{Spp}(\boldsymbol{X})$, where $\boldsymbol{X}:=\left\{\boldsymbol{X}_{r}\right\}_{r \geq 1}$, gives a Poisson superposition process with diagonal measures $\left\{\zeta^{(r)}\right\}_{r \geq 1}$. On the other hand, $N(t):=\sum_{n=1}^{\infty} N^{(n)}(t)$ is a Poisson process with intensity $\lambda$ and

$$
W(t):=\sum_{i=1}^{N(t)} X_{i}, \quad t \geq 0,
$$

determines the paths of a compound Poisson process with intensity $\lambda$ and increment distribution $\left\{p_{k}\right\}_{k \geq 0}$, where $X_{1}, X_{2}, \ldots$ are i.i.d. with $\mathbb{P}\left\{X_{1}=k\right\}=p_{k}$.

5.2.2. Negative binomial processes. Suppose that the $n$th component measure is

$$
\zeta^{(n)}\left(A_{1} \times \cdots A_{n}\right)=\frac{\theta \zeta_{1}\left(A_{1} \cap \cdots \cap A_{n}\right)}{n}
$$

for some finite measure $\zeta_{1}$ on $\mathcal{X}$. Then, each $\zeta^{(n)}$ is diagonal and, from the above discussion, the number of points in nonoverlapping subsets of $\mathcal{X}$ are independent and, for each $A \subseteq X, Z(A)$ is a negative binomial random variable with parameter $\left(\theta, \zeta_{1}(A)\right)$. Note that this definition of negative binomial process differs from Barndorff-Nielsen and Yeo (1969).

\subsection{Boson processes}

A cyclic permutation of $[n]$ is any permutation $\sigma$ with a single cycle. Given a function $M: \mathcal{X} \times \mathcal{X} \rightarrow \mathbb{R}$, we define the sum of cyclic products of $M$ at $x:=\left(x_{1}, \ldots, x_{n}\right)$ by

$$
\operatorname{cyp}(M[x]):=\sum_{\sigma: \# \sigma=1} \prod_{j=1}^{n} M\left(x_{j}, x_{\sigma(j)}\right) .
$$

Suppose now that $\mu$ is a measure on $X$, and that $\mu^{n}$ is the product measure on $X^{n}$. Define $\operatorname{tr}(M)=\int M(x, x) \mathrm{d} \mu(x)$,

$$
M^{2}\left(x, x^{\prime}\right)=\int_{x} M(x, t) M\left(t, x^{\prime}\right) \mathrm{d} \mu(t)
$$


and $M^{k}$ by extension. Let $\zeta=\left\{\zeta^{(n)}\right\}_{n \geq 1}$ be a measure on seq $(X)$ for which the density in $X^{n}$ with respect to $\mu^{n}$ is

$$
\zeta^{(n)}(\mathrm{d} x)=\frac{\zeta_{n}(\mathrm{~d} x)}{n !}=\frac{\alpha \operatorname{cyp}(M[x])}{n !}
$$

for some $\alpha>0$. It follows that $\zeta^{(n)}\left(X^{n}\right)=\alpha \operatorname{tr}\left(M^{n}\right) / n$, so the total measure is

$$
\zeta_{.}=\zeta(\operatorname{seq}(X))=\alpha \sum_{n \geq 1} \frac{\operatorname{tr}\left(M^{n}\right)}{n}=-\alpha \log \operatorname{det}(I-M)
$$

provided that the spectral norm of $M$ is strictly less than 1 . Under positivity conditions, the Janossy measure of the Poisson superposition $Z \sim \operatorname{PSP}(\zeta)$ has a density

$$
\frac{\mathrm{d} J_{n}}{\mathrm{~d} \mu^{n}}(\boldsymbol{x})=\mathrm{e}^{-\zeta .} \sum_{\pi \in \mathcal{P}_{n}} \alpha^{\# \pi} \prod_{b \in \pi} \operatorname{cyp} M(x[b])=\mathrm{e}^{-\zeta \cdot} \operatorname{per}_{\alpha}(M[x]),
$$

which is proportional to the $\alpha$-permanent of $M$ at $\boldsymbol{x}$. Provided that $M$ is symmetric, positive definite, and $2 \alpha$ is a positive integer, this is the Janossy measure of a boson, or permanental, point process.

\section{Acknowledgment}

Harry Crane is partially supported by NSF grant no. DMS-1308899 and NSA grant no. H98230-13-1-0299.

\section{References}

Arratia, R., Barbour, A. D. ANd Tavaré, S. (1992). Poisson process approximations for the Ewens sampling formula. Ann. Appl. Prob. 2, 519-535.

BarndorfF-Nielsen, O. ANd Yeo, G. F. (1969). Negative binomial processes. J. Appl. Prob. 6, 633-647.

Cox, D. R. And Isham, V. (1980). Point Processes. Chapman \& Hall, London.

Crane, H. (2013a). Permanental partition models and Markovian Gibbs structures. J. Statist. Phys. 153, 698-726.

CRANE, H. (2013b). Some algebraic identities for the $\alpha$-permanent. Linear Algebra Appl. 439, 3445-3459.

Donnelly, P. AND GRIMmett, G. (1993). On the asymptotic distribution of large prime factors. J. London Math. Soc. (2) 47, 395-404.

EWEnS, W. J. (1972). The sampling theory of selectively neutral alleles. Theoret. Pop. Biol. 3, 87-112, $240,376$.

Fisher, R. A., Corbet, A. S. AND Williams, C. B. (1943). The relation between the number of species and the number of individuals in a random sample of an animal population. J. Animal Ecol., 12, 42-58.

Hough, J. B., Krishnapur, M., Peres, Y. ANd Virág, B. (2006). Determinantal processes and independence. Prob. Surveys 3, 206-229.

KIngman, J. F. C. (1978). Random partitions in population genetics. Proc. R. Soc. London A 361, 1-20.

Kingman, J. F. C. (1978). The representation of partition structures. J. London Math. Soc. (2) 18, 374-380.

Kingman, J. F. C. (1993). Poisson Processes. Oxford University Press.

MaCCHI, O. (1975). The coincidence approach to stochastic point processes. Adv. Appl. Prob. 7, 83-122.

McCullagh, P. (1987). Tensor Methods in Statistics. Chapman \& Hall, London.

McCullagh, P. and Møller, J. (2006). The permanental process. Adv. Appl. Prob. 38, 873-888.

Pitman, J. (2006). Combinatorial Stochastic Processes (Lecture Notes Math. 1875). Springer, Berlin.

Rubak, E., Møller, J. AND MCCullaGH, P. (2010). Statistical inference for a class of multivariate negative binomial distributions. Res. Rep. R-2010-10, Department of Mathematical Sciences, Aalborg University. 
STREITBERG, B. (1990). Lancaster interactions revisited. Ann. Statist. 18, 1878-1885.

Valiant, L. G. (1979). The complexity of computing the permanent. Theoret. Comput. Sci. 8, 189-201.

VERE-JONES, D. (1988). A generalization of permanents and determinants. Linear Algebra Appl. 111, 119-124.

VERE-JONES, D. (1997). Alpha-permanents and their applications to mulivariate gamma, negative binomial and ordinary binomial distributions. New Zealand J. Math. 26, 125-149.

WILF, H. S. (2006). Generatingfunctionology, 3rd edn. A K Peters, Wellesey, MA. 